\title{
Augmenting Computer Graphics with Haptics for the Visualization of Vessel Networks
}

\author{
Dingrong Yi and Vincent Hayward \\ Department of Electrical and Computer Engineering \& \\ Center For Intelligent Machines \\ McGill University, 3480 University Street, \\ Montreal, Quebec, H3A 2A7 Canada \\ Email: yidingr@cim.mcgill.ca, hayward@cim.mcgill.ca
}

\begin{abstract}
Current visualization methods of volume angiograms are limited in their ability to display vessel connectivity and depth information readily available in the data set. In this work, we introduce a hybrid graphic-haptic display technique that allows visualizing vessel connectivity as well as provides user assistance in tracing vessel branches. Most importantly, our display provides three-dimensional shape cues in both visual and haptic domains that eliminate the need for a rotational display for 3D perception. Issues related to haptic rendering, implementation, and experimental validation of the developed system are reported. This proposed multimodal visualization approach is independent of the devices used and can be applied to current visualization platforms for angiograms due to the small computational load it requires.
\end{abstract}

\section{Introduction}

Techniques such as digital subtracted angiography (DSA), computed topography angiography (CTA), phase contrast magnetic resonance angiography (PCMRI), and time-of-flight magnetic resonance angiography (TOFMRI) provide radiologists and doctors with angiographic images of ever increasing resolutions and signal-to-noise ratios. These improving imaging techniques justify the need for effective visualization methods.

Current available methods of volume angiogram visualization, which include surface rendering, volume rendering, and stereopsis, have not replaced the conventional role of maximum intensity projection (MIP) technique. Continuous rotation is frequently employed to appreciate depth information in a MIP rendered image [27]. Such operation is quite time-consuming for large real volume angiograms, and it imposes heavy computational loads to the computer used in the application. Even with methods for speeding up, the achievable screen update rate can still be limited for large volume images, and this eventually causes the motion depth cue to cease working effectively [23] [33] [5] [6] [7] [17]. In addition, rotational display of the vessel network requires users to mentally rotate their internal representation of the network such that the displayed object can be registered with this representation. However, such mental operation is difficult and time consuming to perform.

In this work, we propose a method that enables users to visualize the shape of a vessel network without having to frequently rotate the object. It provides shape perception even in a static viewing condition between two consecutive rotations. After a user selects desirable viewing parameters, the vessel network and the background tissues essentially remain in a static state, and the only moving object is the computer agent which is similar to a cursor but executes far more functions. The animation path of the agent is along the centerline of vessel branches which can be extracted from raw volume angiogram images [31]. Meanwhile the agent's moving direction and speed are under the user's control through a tracing algorithm. While it is moving, the agent displays depth information by colour cueing and by force feedback. The ultimate goal of this work is to help a user manipulate and interpret large data sets produced by modern imaging techniques.

The rest of the paper is organized as follows: a brief literature review on haptic applications as applied to scientific visualization is in Section 2. The method of user exploration under the guidance of a semi-autonomous computer agent is briefly described in Section 3. A force rendering scheme is elaborated in Section 4. In Section 5, we present the details of our implementation. The results and conclusions of the study are discussed in Sections 6 and 7, respectively. 


\section{Application of Haptic Rendering to Scien- tific Visualization}

Haptic rendering can be defined as the computation of forces sent by a force-reflecting haptic device to the user to convey some information [30].

\subsection{Examples}

One of the first applications of haptic rendering to scientific visualization is the molecular docking system described by Brooks et al. [4] where forces and torques are used to help the user move and orient a drug molecule to an active site of the protein dihydrofolate reductase. The forces and torques are calculated in two stages. The force components are pre-computed at grid points around the active site for probe atoms of each type. Forces and torques are then obtained by vector-summation of force components for each of its atoms in real-time. The authors find that haptic feedback helps a user maneuvre the drug molecule faster and with a shorter trajectory than without it.

Avila and Sobierajski [2] add force feedback to the display of volume images. Forces are calculated according to the opacity of material, which is estimated from the image intensity field and its gradient field. This helps a user appreciate the presence of bones underneath soft flesh. In another application[2], gentle attracting forces are used to reduce the difficulty of tracing the complicated winding dendrites of a lateral geniculate nucleus (LGN) cell. A hardware acceleration method to avoid casting rays through empty regions of the volume is also proposed [25]. This yields a haptic update rate of 1 to $5 \mathrm{kHz}$ for large volume data.

Wang [29] uses force feedback to help a user trace the edges of ultrasound images and other objects. Haptic cues are created as a function of the alignment of the user movement direction with the edge under exploration. McNeely [15] uses 3D forces and torques to help a viewer perceive the 3D nature of a voxelized aircraft and navigate through the congested areas with minimal surface interference. Iwata et al. [12] provide both visual and haptic sensations related to $3 \mathrm{D}$ objects. This is done by projecting the image of an object onto a flexible screen whose height is adjusted to indicate the surface shape of the displayed object. Actuators connecting to the screen with rods return a force as a function of the sensed forces exerted by the user and the rigidity of the object.

In Infed et al. [11], torques are used to display vector fields such as magnetic stream lines. The authors apply restoring torques to force a stylus to align in the direction of the stream line; the torques are proportional to the angle between these two directions.

\subsection{Considerations on Augmenting Graphics with Haptics}

Augmenting a conventional graphic display with a haptic interface is a non-trivial procedure. First, the integrated system is multi-frequency. It is well known that visual displays should update at rates exceeding 20 per seconds in order to produce stable images. For haptics, at the current stage of technological development, it is generally accepted that $1 \mathrm{kHz}$ should be the target update rate. The major reason for such a high update frequency is that humans are quite sensitive to vibrations at a frequency as high as 1000 $\mathrm{Hz}$, although the sensitivity starts to diminish around 300 Hz. Due to sensitivity to vibrations at high frequencies, the sharp transients of force feedback can easily stand out. In extreme cases of low update frequency, the user may experience the force discontinuity at each update. Updating forces at high frequencies can make the sharp transients feel precise, and the integrated system of the human and the haptic device becomes more stable. In addition to the requirement of high update frequency, there is also a constraint on the temporal span between the instant of the user interaction position being sampled and the instant of the consequent force being fed back to the user. This lag should be very short in order to reduce the energy regenerated into the closed-loop system comprising the finger and the held haptic device.

An effective integrated system of haptics and graphics should ensure the effectiveness of each modality. Furthermore, the effectiveness of the system greatly depends on the consistency of the visual and haptic stimuli. The finding by Srinivasan et al. [28] of visual dominance over haptic perception suggests that the haptic feedback should always be consistent with the visual feedback to enhance and complement the visual display. This dominance implicitly requires an unnoticeable lag between the updates of the haptic and visual displays.

\subsection{Methods to Satisfy Multi-frequency Require- ments}

To prevent these two modalities from competing for limited computational resources, several approaches are employed in the existing literature. Mark et al. [14] decouple the haptic servo loop from the main application loop with two separate machines connected through ethernet. The authors use an intermediate representation of the scene which is infrequently updated by the main application but evaluated at a high frequency by the haptic servo loop. They achieved a force feedback update frequency of $1 \mathrm{kHz}$ and provided the feeling of a stiff surface to the user. A second approach to meet the multi-frequency requirement is to reduce the lengthy graphic computations through fast rendering and updating the scene locally around the interaction 


\section{Proc. 10th Pacific Conference on Computer Graphics and Applications October 09 - 11, 2002 Tsinghua University, Beijing pp. 375-384}

position. For example, Avila and Sobierajski [2] successfully employ this approach to the haptic integrated volume visualization system.

It is also desirable to improve the efficiency of the haptic update loop, since it requires to be executed much more frequently than a graphical simulation. An efficient haptic interaction algorithm and/or an efficient storage of data are essential to reduce the execution time of a haptic servo loop. For example, Ho et al. [10] propose their "neighbouring watch algorithm" for efficient haptic contact transitions based on the precomputed proximity information of the data set. Pai and Reissel [19] and Asghar and Barner [1] propose a multi-resolution data representation for $2 \mathrm{D}$ images and 3D images, respectively, for the applications of haptics.

\section{Computer Guided User Exploration on Vessel Systems}

This section briefly describes the graphical component of the integrated system. For more details, please refer to [30].

\subsection{Centerline Reconstruction}

A symbolic representation that consists of one voxelthick centerlines of vessels is a compact form to encode a large 3D volume angiogram [26], [8], [9], [20], [18], [13]. In this work, a symbolic model that approximates centeradjusted shortest lines that connect the two ends of each branch of the vessel network is used. It has the computational advantage of rapid contact transition detection. It also provides accurate geometric features such as tangent, curvature, and torsion at each center point of vessels.

A volume angiogram is first graphically displayed using a MIP technique to provide a global visualization and intuitive understanding of the vessel system (see Figure 9). In MIP methods, the screen pixel intensity is the maximum value encountered by the viewing ray while traveling through the volume image. With a MIP display, a user can manually select a seed point on the vessel system as a reference point. Using forward propagation and voxel coding, a connected and complete network of vessel centerlines can be automatically extracted from binary segmented volume data [32] or from raw intensity volume images with linear operations [31].

To be consistent with the terminology used in [32] and [31], we refer to the centerline representation as the Qskeleton, which is a network of connected paths representing a complete vessel system. Each path contains the following information: a unique path id, a head to indicate where it starts, a tail to indicate where it ends, and a list of spatially-indexed points. Each point on the centerline is referred to by two integers $(p, l)$, where $p$ is the path identifier and $l$ is the point index within that path. Each point on the centerline also contains its geometric location $(x, y, z)$, local orientation $\left(t_{x}, t_{y}, t_{z}\right)$, curvature $c$, and torsion $t$, as well as a bifurcation label and the radius of the cross section.

\subsection{Initial Contact Detection}

We only consider two types of user interaction: the free exploration state and the tracing state (see Figure 1). A graphic cursor is used to reflect the user's actual position; its relation to a haptic device is the same as that of a cursor to a mouse. During exploration, proximity of the cursor to a vessel terminates this state and triggers the tracing state. A button click returns the user to the exploration state from the tracing state.

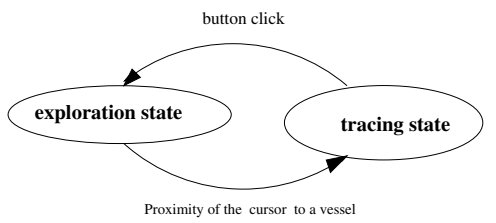

Figure 1. Two states of a user's interactions with the vessel network.

The initial contact location is found from a look-up table indexed by the 2D locations of the cursor. One table entry corresponds to one pixel. Two integers are stored, indicating that the pixel is either occupied by a point $(p, l)$ on the Q-skeleton or is empty with negative integers. At each time step, the 2D location of the cursor retrieves the corresponding integers by which the contact information is readily available. When the cursor is on or close enough to a point $(p, l)$ on the Q-skeleton, an initial contact occurs there and the interface switches from exploration to tracing. This 2D method of contact detection takes advantage of the Q-skeleton where little occlusion occurs.

\subsubsection{Tracing Algorithm}

During the tracing state, the cursor becomes invisible and a computer agent is activated. This agent moves in $3 \mathrm{D}$ and is constrained on the vessels' centerline. It is displayed as a $3 \mathrm{D}$ cone to visually indicate its movement direction along the local vessel orientation.

At each time step, the cursor-movement vector is constituted to start at the previous location and to end at the updated location. The agent automatically locates itself on the vessel centerline according to this vector. At a location $(p, l)$, the agent has only two potential movement directions if the vessel does not bifurcate: to the left (decreasing indices) or to the right (increasing indices). If the vessel bifurcates at point $(p, l)$, the agent has an additional moving 


\section{Proc. 10th Pacific Conference on Computer Graphics and Applications October 09 - 11, 2002 Tsinghua University, Beijing pp. 375-384}

direction, which is towards the first point of the bifurcating branch $\left(p^{\prime}, 0\right)$ if the path $p^{\prime}$ connects the path $p$ at point $l$. The agent determines its direction to be the one corresponding to the largest vector projection on the centerline. If the projection magnitude is below a threshold, the agent remains stationary during this time step. The traveling distance is iteratively determined from the arc length of the vessel subtended by the vector projection.

\subsection{Graphic Depth Cue}

The computer agent is graphically displayed as a 3D cone, colored red at the apex and sides and blue at the base. The cone is displayed at the user interaction location and is oriented along the local tangent vector of the vessel pointing towards its movement direction. When the cone is coming towards the user in depth direction, its blue base is invisible and it appears red. On the contrary, when it is moving away from the user, it presents its blue base partially or completely to the user. When there is no depth change along the trajectory of the proxy, the cone looks like a red triangle. In this way, the cone indicates the local orientation of the vessel related to the projection plane (See Figure 2).
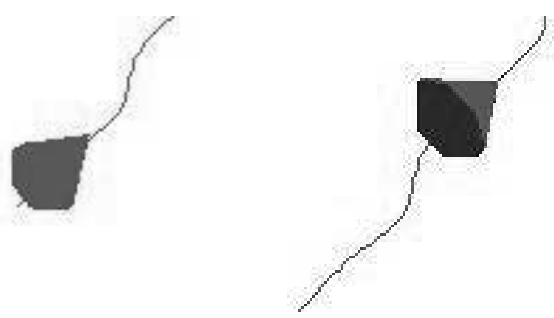

Figure 2. The left panel shows that the agent is coming towards the viewer and the right shows that it is going away in depth from the viewer.

\section{Force Rendering Scheme}

2D haptic feedback is provided to the user as a force in a plane. This force is equivalent to the lateral component of the force applied to a point mass sliding on a frictionless vessel centerline by a uniform gravity field. It is described in terms of infinitesimal quantities like $d l$ and $d z$, but computationally, these quantities correspond to small incremental values like $\Delta l$ and $\Delta z$ measured during two consecutive time steps. The two dimensional force is described by:

$$
F=k(P) \frac{d z}{d l} \eta
$$

Referring to Figure 3, $d z$ is the depth difference during the travel of the mass along a vessel segment, while $d l$ is the projected length of the mass displacement on the viewing plane. The ratio $\frac{d z}{d l}$ then is the local "depth gradient" of the vessel centerline. The vector $\eta$ is a unit vector contained in the view plane. It is tangent to the projected centerline at the point of interaction in the viewing plane. The scalar function $k(P)$ depends on the magnitude $P$ of the vertical component of the force exerted by the user on the haptic device. The force generated by equation 1 is called the lateral depth gradient force (LDGF), a force in two dimensions.

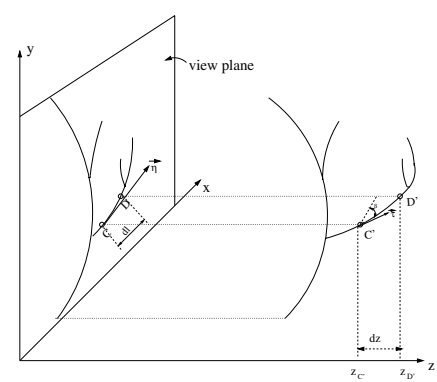

\section{Figure 3. Perspective projection of the vessel network.}

The returned force either helps or hinders the user's movement with a magnitude proportional to the movement of the mass in the depth direction and according to the direction of exploration. The faster $z$ increases (decreases), the stronger the user's hand is pushed (pulled).

\subsection{Gradient Singularities}

At some locations, the smoothed skeleton can be parallel to the viewing direction and yields an infinite depth gradient due to $d l$ vanishing. The force generated by equation 1 becomes unbounded and the scheme breaks down. If left untreated, the singularity may cause a number of unexpected results. Rather than clamping the intensity of $F$, the force function is regularized by adding a small constant parameter $\lambda$ to the denominator. The force generated by equation 2 still reflects the gradient of the varying depth:

$$
F=k(P) \frac{d z}{d l+\lambda} \eta
$$

\subsection{Projection Discontinuities}

Force discontinuities, either in magnitude or in direction, must be avoided. An occasional large change in the depth value will cause a force magnitude transient. An object with infinite-order continuity commonly lacks first-order continuity when projected in the viewing plane in some viewing 
directions. Figure 4 illustrates how view transformations cause a discontinuity problem. It shows a cylindrical spiral as projected on the screen.

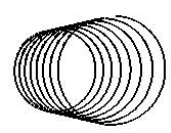

(a)

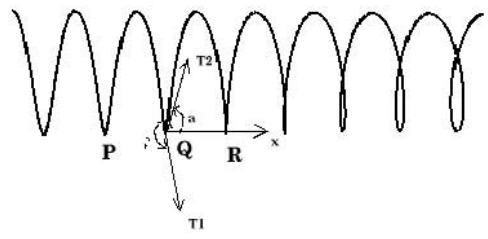

(b)
Figure 4. An illustration of one kind of discontinuity problem which results from view transformation (a) a spiral, (b) a perspective projection of the spiral which contains sharp corners at $Q$ and $R ; \alpha$ and $\beta$ are the angles of the local tangents at position $Q^{+}$and $Q^{-}$ relative to the $x$-axis.

The force direction is along the tangent of the projected curve. At time instant $t_{1}$, the force is along tangent $\vec{T}_{1}$; immediately after, at instant $t_{2}$, it is along tangent $\vec{T}_{2}$. Assuming that the force magnitude remains almost constant between $t_{1}$ and $t_{2}$, the relative changes of $f_{x}$ and $f_{y}$

$$
\begin{aligned}
& \frac{\delta f_{x}}{f_{x}}=-\tan \left(\frac{\alpha+\beta}{2}\right) \tan \left(\frac{\alpha-\beta}{2}\right) \\
& \frac{\delta f_{y}}{f_{y}}=-\cot \left(\frac{\alpha+\beta}{2}\right) \tan \left(\frac{\alpha-\beta}{2}\right)
\end{aligned}
$$

monotonically increase with $\tan \left(\frac{\alpha-\beta}{2}\right)$. When $\alpha$ and $\beta$ are close to or equal to each other, the force change is smooth; however, when $\alpha-\beta$ is close to $\pi$ (for example, near point $Q$ in figure $4 \mathrm{~b}$ ), the force change becomes infinite, or otherwise very large.

One approach to solve this problem is to clamp the force increments. But, this is not a good approach because the force that renders the shape becomes severely distorted. Instead, large increments required during one time step are produced by several small ones during a few time steps (see figure 5). In other words, large force steps are smoothed in the time domain rather than in the spatial domain.

\subsection{Interpretation of the Lateral Depth Gradient Force}

As can be seen from the above, the user actually moves in a plane, and the forces experienced are two-dimensional. It may seem counter-intuitive that the experienced sensation is the geometry of a three-dimensional shape. Consider an object that is sliding on a frictionless surface (see Figure 6);
// This is done at the final stage of force generation proc force interpolation

$$
\begin{aligned}
& \mathrm{n}:=\operatorname{maxi}\left(\frac{f_{x}\left(t_{2}\right)-f_{x}\left(t_{1}\right)}{\text { threshold }}, \frac{f_{y}\left(t_{2}\right)-f_{y}\left(t_{1}\right)}{\text { threshold }}, 1\right) ; \\
& \delta f_{x}=\frac{f_{x}\left(t_{2}\right)-f_{x}\left(t_{1}\right)}{n} \\
& \delta f_{y}=\frac{f_{y}\left(t_{2}\right)-f_{y}\left(t_{1}\right)}{n} \\
& \text { for } i:=1 \text { to } n \text { do } \\
& \quad f_{x}\left(t_{i}\right)=f_{x}\left(t_{i-1}\right)+\delta f_{x} \\
& \quad f_{y}\left(t_{i}\right)=f_{y}\left(t_{i-1}\right)+\delta f_{y} \\
& \text { od }
\end{aligned}
$$

Figure 5. An outline of a force interpolation algorithm showing how each large increase in forces is generated as the sum of several finer increments over a number of time steps.

the slope of the surface in the direction of movement is related to the lateral force $f$ that drives it downwards. The simulated forces we provide to the user would be equivalent to the force $f$ exerted on the object. In our simulation, we use the product of a factor $k(P)$ which depends on the pushing force $P$ exerted by the user on the stylus to replace the force $N$ that the surface applies to the object. The lateral depth gradient force model provides a correct sensation of shape. This may be due to our natural response to gravity, which defines what is "up" and what is "down".

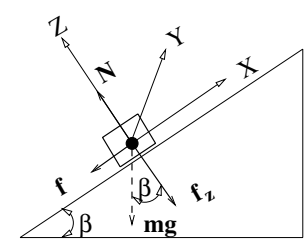

Figure 6. An illustration of the forces exerted no an object with mass $m$ on a frictionless surface with slope $\tan \beta$. The object responds to the slope of the surface by the amount of tangential driven force $f$.

The depth gradient force scheme is different from the conventional surface gradient force used to generate the haptic sensation of surface texture [16]. The local surface gradient $\left(\frac{\partial z}{\partial x}, \frac{\partial z}{\partial y}\right)$ is independent of direction of movement. In contrast, a force associated to the slope in the direction of movement provides information that depends on exploration. 


\subsection{Depth Gradient Force vs Surface Gradient Force}

The conventional lateral gradient force is commonly used to synthesize surface textures and roughness [16], [24], [10], [3]. However, the depth gradient force proposed here is a better indicator for spatial variations of curves and surfaces than the lateral gradient force.

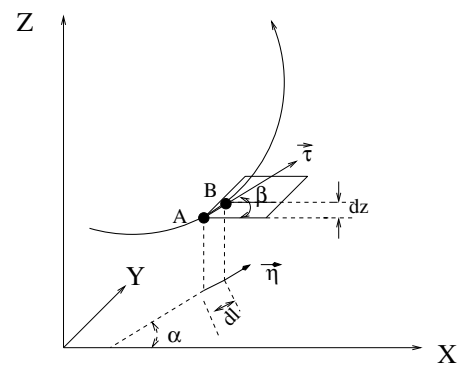

Figure 7. An illustration to define the mathematical symbols used in the text. $\vec{\tau}$ is a three-dimensional unit vector that is tangent to a vessel segment on the skeleton at an interaction location A. $\vec{\eta}$ is a two-dimensional unit vector in the viewing plane determined by projecting $\vec{\tau}$ onto the $x y$-plane. $\alpha$ is the angle between $\vec{\eta}$ and the $x$-axis, and $\beta$ is the angle between $\vec{\tau}$ and the $x y$-plane.

Consider two nearby points $A$ and $B$ on a three dimensional curve (see Figure 7). The arc-length that separates them is approximately represented by the length of a short line segment $\bar{A} \bar{B}$. This segment defines an angle $\beta$ with respect to the $x y$ plane and $\alpha$ with respect to the $x$-axis. The lateral depth gradient force ${ }^{d} \vec{F}=\left({ }^{d} F_{x},{ }^{d} F_{y}\right)$ is (the factor $k$ is dropped here):

$$
\begin{aligned}
\overrightarrow{{ }^{d} F} & =\left(\frac{\delta z}{\delta l}\right) \vec{\eta} \\
& =\left(\frac{\delta z}{\delta l} \cos \alpha, \frac{\delta z}{\delta l} \sin \alpha\right) \\
& =\left(\frac{|\bar{A} \bar{B}| \sin \beta \cos \alpha}{|\bar{A} \bar{B}| \cos \beta}, \frac{|\bar{A} \bar{B}| \sin \beta \sin \alpha}{|\bar{A} \bar{B}| \cos \beta}\right) \\
& =(\tan \beta \cos \alpha, \tan \beta \sin \alpha)
\end{aligned}
$$

where

$$
\begin{array}{r}
\quad \delta z=|\bar{A} \bar{B}| \sin \beta \\
\delta x=|\bar{A} \bar{B}| \cos \beta \cos \alpha \\
\delta y=|\bar{A} \bar{B}| \cos \beta \sin \alpha
\end{array}
$$

$$
\delta l=\sqrt{(\delta x)_{2}+(\delta y)_{2}}=|\bar{A} \bar{B}| \overrightarrow{c o s} \beta
$$

The lateral gradient force would give:

$$
\begin{aligned}
\overrightarrow{{ }^{g} F} & =\left(\frac{\partial z}{\partial x}, \frac{\partial z}{\partial y}\right) \\
& =\left(\frac{|\bar{A} \bar{B}| \sin \beta}{|\bar{A} \bar{B}| \cos \beta \cos \alpha}, \frac{|\bar{A} \bar{B}| \sin \beta}{|\bar{A} \bar{B}| \cos \beta \sin \alpha}\right) \\
& =\left(\frac{\tan \beta}{\cos \alpha}, \frac{\tan \beta}{\sin \alpha}\right)
\end{aligned}
$$

The depth gradient force in equation 3 and the gradient force in equation 4 are both proportional to the local slope, $\tan \beta$. However, they differ in other aspects. The depth gradient force is along the movement direction, while the gradient force is not. In addition, when $\alpha$ is $90^{0}$ or $0^{0}$, the gradient force would have an infinite magnitude and create force discontinuities.

The projected conventional gradient force onto the direction of the proxy movement $\vec{\eta}=(\cos \alpha, \sin \alpha)$ coincides with the lateral depth gradient force:

$$
\begin{aligned}
\left(\overrightarrow{{ }^{g} F} \bullet \vec{\eta}\right) \vec{\eta} & =\left(\frac{\tan \beta}{\cos \alpha}, \frac{\tan \beta}{\sin \alpha}\right) \bullet(\cos \alpha, \sin \alpha) \vec{\eta} \\
& =\tan \beta \vec{\eta} \\
& =\overrightarrow{{ }^{d} F}
\end{aligned}
$$

\subsection{Haptic Devices}

Any haptic device that is capable of measuring position and generating force output in two or more dimensions can be used. Possible haptic devices include, but are not limited to, the Pantograph [21], the PenCat/Pro ${ }^{\mathrm{TM}}$ by Immersion Canada, the Phantom by Sensible Technologies, Inc., or the XTerminator force-feedback game pad by Gravis.

\section{Implementation}

The rendering method described in Section 3 and 4 is included in a multi-modal visualization platform. The hardware components of the setup include a personal computer (PC) and a PenCat/Pro ${ }^{\mathrm{TM}}$ haptic device (Immersion Canada Inc.). The PC runs the visualization toolkit (VTK) to graphically display the volume angiogram. The PenCat/Pro ${ }^{\mathrm{TM}}$ haptic device allows the user to experience the force sensations while manually exploring the vessels. To provide a satisfactory experience, the graphic and haptic update rates need to be maintained at around or above $45 \mathrm{~Hz}$ and $1000 \mathrm{~Hz}$, respectively. 


\section{Proc. 10th Pacific Conference on Computer Graphics and Applications October 09 - 11, 2002 Tsinghua University, Beijing pp. 375-384}

\subsection{Software Architecture}

Multiprocessing was used to synchronize the visual display refresh cycle and the haptic update loop. Figure 8 illustrates these functions and indicates the execution frequency of each of the processes. The processes communicate through several first-in-first-out (FIFO) buffers. The haptic process is designed to be small and computationally simple in order to achieve a high rate. It holds in shared memory the view-transformed centerline representation of the vessel network instead of the large-sized full volume angiogram. Other tasks are: sampling of the user position, detection of contact transition, and force update. They are grouped into the haptic loop because of the necessity for these tasks to have both a high time resolution and a high execution frequency.

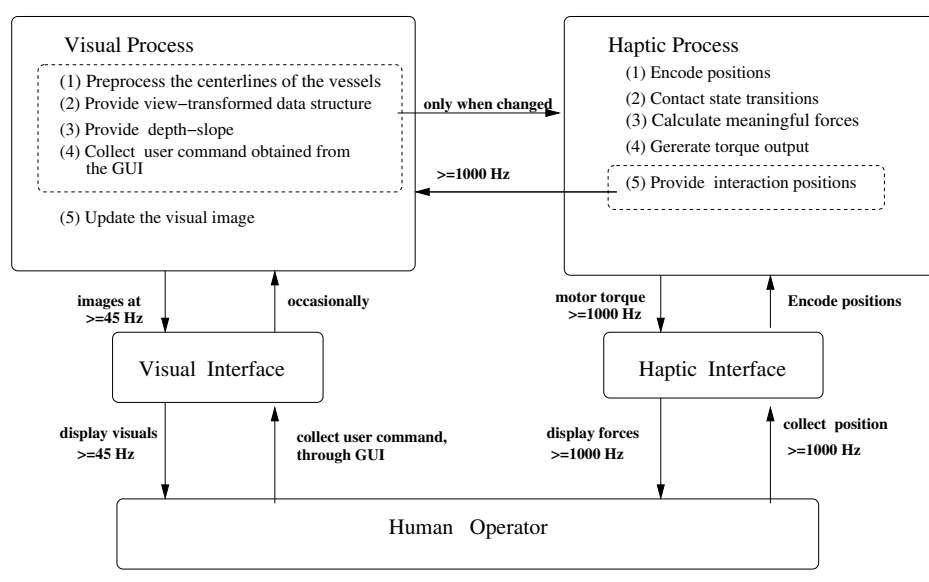

\section{Figure 8. A multiprocessing software archi- tecture for the integration of haptic and graphic modalities.}

The actual force calculation is carried out in two stages. The first stage determines the depth gradient at each point of the vessel centerline, which depends on the view parameters and, thus, is placed in the visual loop. The second combines the depth gradient and user movement and places them in the haptic loop which runs at a higher frequency.

Other tasks that can be executed less frequently are grouped into the visual process. These include the centerline extraction which needs to be done only once and is performed in pre-processing, the view transformation of the full angiogram and of the centerlines, the collection of user commands through a GUI, and the update of the graphic representation of the agent and of the full vessel network. Only the pixels that have changed since the last update are modified. Updating the full screen would be too time consuming with a MIP display or a more general volume rendering of the angiogram.

\subsection{Toward a User-Friendly Interface}

There are two control modes associated with the $z$ input of the haptic device: the re-indexing mode and the forcereflecting mode. Taking the PenCat $/ \mathrm{Pro}^{\mathrm{TM}}$ as an example, the re-indexing mode is triggered when the user lifts the tip of the stylus, just like lifting a mouse to enter its re-indexing mode. While in this mode, user's hand movement leaves the cursor stationary. Users use re-indexing to maintain a comfortable working position as if the haptic device had a relative working space. The concept of haptic re-indexing is useful for any haptic application where the interaction position is subject to workspace constraint.

The force-reflecting mode depends on the $z$ movement of the stylus when it is pushed downwards. There are 3 zones. A zero-force zone prevents any activity when the device is left unattended. In the next zone, the force increases with the $z$ value: the harder the user pushes, the larger the forces are. It is in this zone that the user is advised to move the stylus of the haptic device to explore shapes. In this zone, $k(P)$ in equation 2 is a function of the vertical component of forces that the user exerts on the stylus. The next zone is the saturation zone. In this zone, the downward movement of the stylus is limited and $k(P)$ reaches its maximum.

Another concept that was introduced is the concept of haptic zoom. The graphic/haptic ratio $r$ defined as the ratio between the movement of the cursor on the graphic window and the the actual user movement of the stylus can be adjusted using a sliding bar. When fine control is desired to enhance the details of an object, the user adjusts $r$ to a value smaller than one. A large movement of the user's hand then corresponds to a small movement of the pointer on the screen. This "haptic magnification" is also useful for exploring small objects displayed together with larger ones. It would be interesting to carry out a psychological experiment to determine optimum graphic/haptic ratios.

\section{Examples}

\subsection{Synthetic Example}

The multimodal visualization system was first applied to some known artificial objects such as a circle, a winding line oscillating in a plane, and a spiral in order to tune and debug the system.

When a circle is oriented such that it is viewed as a narrow oval, the force sensation conveys two different types of information, namely that it is rounder than it appears and which side is in front. When the plane which contains the winding line lies in parallel to the viewing direction, it appears as a straight line. The presence of hills and valley is made obvious haptically, however. 


\section{Proc. 10th Pacific Conference on Computer Graphics and Applications October 09 - 11, 2002 Tsinghua University, Beijing pp. 375-384}

\subsection{Actual Example}

The system was used to navigate in a real volume angiogram. Its size is $360 \times 330 \times 420$ voxels with a spatial resolution of $0.54 \times 0.54 \times 0.54 \mathrm{~mm}^{3}$ (see Figure 9). The angiogram is seen as a MIP display to provides the general structure of the vessel network. Although the displayed vessel network is much more complex than the previous artificial objects, the visual image can still be updated at $45 \mathrm{~Hz}$ and the haptic force feedback can be provided to the viewer at a frequency as high as $2 \mathrm{kHz}$. At such update frequencies, the agent is able to indicate the interaction position without any perceptible delay, and the forces provided to the user are very smooth.

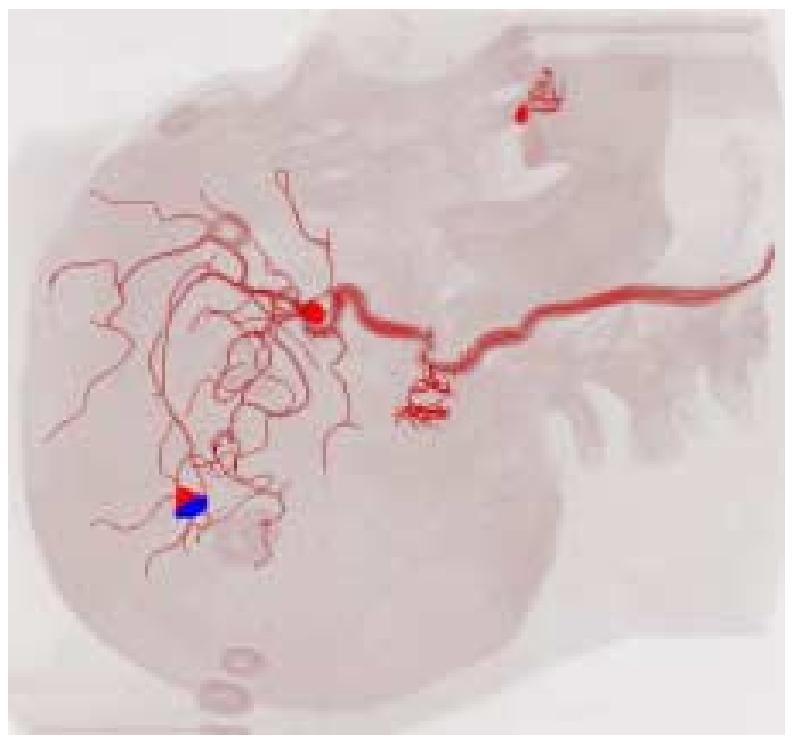

Figure 9. The visual display of the vessel network in the multimodal navigation system. The little cone is the agent which indicates the user-interaction position on the vessel.

\subsection{Human Performance Experiments}

A preliminary experiment was conducted to practically evaluate the usefulness of the proposed method to indicate the shape of vessels. In this paper, only human performance on depth perception is evaluated.

\subsubsection{Subjects, Task, and Procedure}

Four unpaid McGill University students including the first author participated in the experiment. The task was to discriminate the depth relationship between two separate dots on the cerebral vessel and to decide which one was closer to the viewer. The two dots were colored red and green. The haptic stimuli were the forces by equation 2 provided to the viewer as feedback while (s)he explored the vessels. The visual stimuli was the graphic cue sliding on the centerlines of vessels without the MIP display so that the subjects were unaware of the purpose of the experiment.

Before the experimental trials, a subject was trained until (s)he accomplished the following: freely navigating the 3D cone with the haptic device on an artificial object, knowing how to input his/her decision, and finally, building his/her decision-making confidence with the feedback provided after each training trial. The length of a general training session was between 10 to 30 minutes. The object used for experimental trials was the real vessel network, not the one used during the training session to avoid any recall of the shape. All subjects were informed that their decision and the time they took to make them would be automatically recorded by the computer. They were encouraged to proceed quickly and accurately. Meanwhile, they were informed of the importance of accuracy over speed.

\subsubsection{Experimental Results}

Each of the participants completed all of the 102 experimental trials. The mean accuracy was calculated as the ratio of the number of trials with correct responses over the number of all trials and was equal to $99.2 \%$, with a standard deviation $\sigma_{\text {accuracy }}=21 \%$. The mean decision-making time was 5.4 seconds with a standard deviation $\sigma_{\text {time }}=4.9$ seconds. Trials with a flatter depth slope were harder and took longer to discriminate than ones with a steeper depth slope; trials with sharp turning corners took longer to navigate than trials with straighter curve segments. None of the subjects complained about any visual or hand fatigue.

\section{Discussion and Conclusion}

In this work, the display of volume angiograms is conducted locally at the user interaction location by a computer agent in two domains. The agent displays depth visually by colour cueing. Because the cone always points towards its movement direction, the visibility of the blue base indicates whether it is leaving or coming towards the user in the depth direction. A new force model based on depth change intuitively displays the shape of vessel networks. The force magnitude is proportional to the change of depth of the vessel, and its direction is in alignment with the local tangent of the vessel. Thus, the forces provide not only the depth cues but also the local orientation of the vessel centerline. Over time, the continuous force vectors provide sensations of curvatures. The torsion can be provided to the user graphically by a rotation of the agent along the local tangent of the vessel centerline. Although these geometrical quantities of the 


\section{Proc. 10th Pacific Conference on Computer Graphics and Applications October 09 - 11, 2002 Tsinghua University, Beijing pp. 375-384}

shape are displayed locally at the location of the agent, this is not a drawback of the proposed technique. This is due to the fact that the moving agent at the user-interaction position can catch the user's attention, and through a sequential exploration of the vessel, the user can mentally build, over time, a representation of a longer-lasting shape. The haptic cue alleviates the visual channel already overloaded by the features of graphic display. The user can use either the graphic or the haptic cues at any time or use both simultaneously.

One advantage of the proposed technique is that it allows the user to "feel" accurately and efficiently the geometrical shape through active touch [22]. Other advantages are the lightness of the required computational load, its effectiveness for people with bad vision and/or color blindness, and its independence of the haptic device used. It can be rendered by any haptic device which can provide position inputs of two or more degrees of freedom and generate two-dimensional forces as output. Furthermore, it reduces the vision fatigue that is generally caused by graphic-shape cues.

This multimodal visualization technique can be integrated with the current angiogram visualization platforms of radiologists and doctors. Users are able to manually explore the vessel network to perceive its geometrical shape with the force sensations. The semi-autonomous agent keeps the user's work load to a minimum during the exploration/visualization process by assisting him/her in tracing the branches of the vessel network and by providing the forces it would experience while climbing and falling.

\section{References}

[1] M. W. Asghar and K. E. Barner. Nonlinear multiresolution techniques with applications to scientific visualization in a haptic environment. IEEE Transactions on Visualization and Computer Graphics, 7(1):76-93, January-March 2001

[2] R. S. Avila and L. M. Sobierajski. A haptic interaction method for volume visualization. IEEE Proceedings of Visualization 96', pages 197 - 204, 1996.

[3] C. Basdogan, Ho. C. H., and M. A. Srinivasan. A raybased haptic rendering technique for displaying shape and texture of 3D objects in virtual environments. In Proceedings of the ASME Dynamic Systems and Control Division, ASME 1997, volume DSC-Vol.61, pages 77-84, 1997.

[4] F. P. Brooks, M. Ouh-Young, J. J. Batter, and P. J. Kilpatrick. Project grope haptic displays for scientific visualization. ACM SIGGRAPH Computer Graph- ics, Conference proceedings on Computer graphics, 24(4):177-185, September 1990.

[5] W. Cai and G. Sakas. Maximum intensity projection using splatting in sheared object space. In Proceedings EUROGRAPHICS'98, pages C113 - C124, 1998.

[6] B. Csebfalvi, A. Konig, and E. Groller. Fast maximum intensity projection using binary shear-warp factorization. In Proceedings of the 7-th International Conference in Central Europe on Computer Graphics, Visualization and Interactive Digital Media'99, pages 47 $-54,1999$.

[7] W. Heidrich, M. McCool, and J. Stevens. Interactive maximum projection volume rendering. In Proceedings Visualization' 95, pages 11 - 18, 1995.

[8] C. J. Henri and T. M. Peters. Three-dimensional reconstruction of vascular trees: experimental evaluation. Medical Physics, 23(6):617 - 627, 1996.

[9] C. J. Henri and T. M. Peters. Three-dimensional reconstruction of vascular trees. theory and methodology. Medical Physics, 23(2):197, February 1996.

[10] C. H. Ho, C. Basdogan, and M. A. Srinivasan. Efficient point-based rendering techniques for haptic display of virtual objects. Presence, 8(5):477 - 491, 1999.

[11] F. Infed, S. V. Brown, C. D. Lee, D. A. Lawrence, A. M. Dougherty, and L. Y. Pao. Combined visual/haptic rendering modes for scientific visualization. In Proceedings of the 8th Annual Symposium on Haptic Interfaces for Virtual Environment and Teleoperator Systems, November 1999.

[12] H. Iwata, H. Yano, F. Nakaizumi, and R. Kawamura. Project feelex. In Proceedings of the 2001 conference on Computer Graphics August 2001, pages 469-476, August 2001.

[13] K. Krissian, G. Malandain, N. Ayache, R. Vaillant, and Y. Trousset. Model based detection of tubular structures in 3D images. Technical Report 3736, Institut National De Recherche En Informatique Et En Automatique, 1999.

[14] W. R. Mark, S. C. Randolph, M. Finch, J. M. V. Verth, and II. R. M. Taylor. Adding force feedback to graphics systems: Issues and solutions. In Computer Graphics Proceedings, Annual Conference Series, pages 447-452, 1996. 


\section{Proc. 10th Pacific Conference on Computer Graphics and Applications October 09 - 11, 2002 Tsinghua University, Beijing pp. 375-384}

[15] W. A. McNeely, K. D. Puterbaugh, and J. J. Troy. Six degree-of-freedom haptic rendering using voxel sampling. In Proceedings of the SIGGRAPH 1999 annual conference on Computer graphics, pages 401409, 1999.

[16] M. Minsky and S. J. Lederman. Simulated haptic textures: Roughness. In K. Danai, editor, Proceedings of ASME Dynamic Systems and Control Division, volume 58, pages 421 - 426. ASME International Mechanical Engineering Congress Exposition, 1996.

[17] L. Mroz, H. Hauser, and E. Groller. Interactive high-quality maximum intensity projection. Computer Graphics Forum, 19(3), 2000.

[18] I. Nystrom. Skeletonization applied to magnetic resonance angiography images. In K. M. Hanson, editor, Medical Imaging 1998: Image Processing, Proc. SPIE 3338, pages $693-701,1998$.

[19] D. k. Pai and L. M. Reissell. Touching multiresolution curves. In K. Danai, editor, Proceedings of ASME Dynamic Systems and Control Division, volume 58 of DSCD, pages 427 - 432. ASME International Mechanical Engineering Congress and Exposition, 1996.

[20] A. Puig, D. Tost, and I. Navazo. An interactive cerebral blood vessel exploration system. In 1997 IEEE Visualization Conference, pages 443 - 446, 1997.

[21] C. Ramstein and V. Hayward. The pantograph: a large workspace haptic device for a multi-modal humancomputer interaction. In $\mathrm{CHI}^{\prime} 94$, Conference on $\mathrm{Hu}$ man Factors in Computing Systems ACM/SIGCHI, Boston, MA, April 1994.

[22] G. Robles-De-La-Torre and V. Hayward. Force can overcome object geometry in the perception of shape through active touch. Letters to Nature, 412:445-448, July 2001.

[23] G. Sakas, M. Grimm, and A. Savopoulos. Optimized maximum intensity projection. In Proceedings of 5th EUROGRAPHICS Workshop on Rendering Techniques, pages 55 - 63, Doublin, Ireland, 1995.

[24] Y. Shi and D.K. Pai. Haptic display of visual images. In IEEE Annual Virtual Reality International Symposium, pages 188-191, 1997.

[25] L. M. Sobierajski and R. S. Avila. A hardware acceleration method for volumetric ray tracing. In Proceedings of the 6th IEEE Visualization Conference (VISUALIZATION '95), volume 6, pages 27-34, 1995.
[26] R. L. Sollenberger and P. Milgram. A comparative study of rotational and stereoscopic computer graphic depth cues. In Visions : 35th annual meeting: proceedings of the Human Factors Society 35th Annual Meeting / sponsored by the Bay Area Chapter., San Francisco, California, September 1991. Santa Monica, CA : Human Factors Society.

[27] R. L. Sollenberger and P. Milgram. Effects of stereoscopic and rotational displays in a three-dimensional path-tracing task. Human Factors, 35(3):483-499, 1993.

[28] M. A. Srinivasan, G. L. Beauregard, and D. L. Brock. The impact of visual information on the haptic perception of stiffness in virtual environments. In Proceedings of the ASME Dynamics Systems and Control Division, volume DSC-Vol. 58, pages 555 - 559, 1996.

[29] Q. Wang. Translation of graphic to haptic boundary representation. Master's thesis, McGill University, 1999.

[30] D. Yi. Computer Aided Display of 3D angiograms, Using Graphics and Haptics. PhD thesis, McGill University, Department of Electrical \& Computer Engineering, 3480 University Street, McConnell Building, Montreal, Quebec, H3A 2A7, Canada, June 2002.

[31] D. Yi and V. Hayward. Linear cost reconstruction of vascular trees from intensity volume angiograms. In Proceedings of the Second International Conference on Image and Graphics, 2002. Accepted.

[32] D. Yi and V. Hayward. Skeletonization of volumetric angiograms for display. Computer Methods in Biomechanics and Biomedical Engineering, 2002. Accepted.

[33] Viergever Zuiderveld, Koning. Techniques for speeding up high-quality perspective mip. Pattern Recognition Letters, 1994. 